\title{
Adverse Drug Reactions to Anti-TB Drugs: Pharmacogenomics Perspective for Identification of Host Genetic Markers
}

\author{
Kamal Kishor ${ }^{1}$, Roshan Kumar Sahu ${ }^{2}$ \\ ${ }^{1}$ Institute of Pesticide Formulation Technology, India, ${ }^{2}$ CSIR Delhi, India
}

Adverse drug reactions (ADRs) are associated with clinical morbidity and, in severe cases, even mortality. Globally billions of dollars are spent on managing these ADRs for common and uncommon diseases. The developing world suffers from a high burden of tuberculosis, which requires 6-8 months of multi-drug treatment. In spite of most cases being treatable the problem persists mainly due to a high attrition rate associated with ADR mediated complications. Due to these reasons drug resistant strains have emerged and are now a serious challenge to TB eradication. To effectively deliver the available treatment regimen and ensure patient compliance it is important to manage ADRs more efficiently. Recent studies have demonstrated that drug outcomes are patient-specific and can, therefore be predicted. A few of these drugs, including a few administered for TB, have shown excellent correlation with response rates and development of ADRs. In this review, we profile information available in public domain for existing anti-TB drugs to understand the genesis of ADRs and patient response. Additionally, human genome variation databases have been used to correlate the frequency of these markers and their genomic variants in different populations. 10 genes identified which maybe directly linked to ADRs to various anti-TB drugs, 4 of these have been documented earlier. Nearly 47 genes were identified for indirect association with ADRs by virtue of them being off-targets of the drugs. 5 genes were reported for their allelic association with anti-TB DIH. 